\title{
A unified framework for adaptive inverse power control
}

\author{
Marko Höyhtyä* (D) and Aarne Mämmelä
}

\begin{abstract}
In this paper, a unified framework for adaptive inverse power control is developed. It is based on a modified filtered-x least mean square (MFXLMS) algorithm that is proposed and analyzed. A practical version of the algorithm for closed loop power control is also developed. The filtered-x least mean square (FxLMS) algorithm is widely used for inverse control such as noise cancelation. This is the first paper to apply the algorithm for power control. We have modified the conventional FxLMS algorithm by adding absolute value blocks since power control does not need phase information. The modification makes the algorithm more robust and requires fewer bits to be transmitted in the feedback link. The main contribution of the paper is that the proposed algorithm can be seen as generalized inverse control to be used in power control research. It gives a unified framework for several existing algorithms, linking them to the least mean square (LMS) literature. Numerical results are provided, comparing the performance of the proposed algorithm to existing practical algorithms used, e.g., in Third Generation Partnership Project (3GPP) long-term evolution (LTE) systems.
\end{abstract}

Keywords: Power control, Feedback control systems, Adaptive signal processing

\section{Introduction}

Inverse control has been used for several applications such as channel equalization $[1,2]$ automatic gain control (AGC) [3], noise and interference cancelation [4, 5], and transmission power control $[6,7]$ which is the topic of this paper. Due to stability problems, the least mean square (LMS) algorithm is not directly suitable for active control applications where the adaptive filter works as a controller for a time-variant system. Instead, the filtered$x$ least mean square (FxLMS) algorithm is a good choice for that kind of applications [4]. It is essentially the LMS algorithm with a few little changes so that algorithm can remain stable. The FxLMS algorithm is developed from the LMS algorithm by inserting the model of the controlled system between the input data signal and the adaptive algorithm that updates the coefficients of adaptive filter. The algorithm was introduced independently in $[8,9]$ and [10] for adaptive control and noise cancelation.

Conventionally, power control research and LMS algorithm research have been advanced in separate paths. We propose and demonstrate a new use of the FxLMS

\footnotetext{
* Correspondence: marko.hoyhtya@vtt.fi

VTT Technical Research Centre of Finland Ltd, P.O. Box 1100, FI-90571 Oulu, Finland
}

algorithm in this article, namely power control. This integrates previously separated paths, providing unified framework for adaptive inverse power control.

We described the FxLMS method initially for power control in [11] and compared it by numerical simulations to other practical algorithms in [11] and [12]. We developed also a truncated version of the algorithm in [13] to improve energy efficiency. Truncation means that the transmission is interrupted and transmission power is zero when the magnitude of the channel gain deteriorates under a certain cutoff value. Since transmission power control is a new application for the algorithm, new phenomena occur and modifications are needed. Fading in the wireless channel has a wide dynamic range, and changes are fast compared to conventional control systems. In addition, wireless feedback channel limits the number of bits used in control commands. We have modified the conventional FxLMS algorithm by adding absolute value blocks since power control does not need phase information. The modification makes the algorithm more robust and requires fewer bits to be transmitted in the feedback link.

In this paper, we show with analysis that the proposed algorithm converges exactly to the wanted solution in a

\section{Springer}


noiseless channel. We restrict our investigation purely to the closed loop part, focusing on the algorithm and thus assuming ideal feedback. Simulations show that the algorithm converges well also in a noisy channel. The main contribution of this paper compared to our previous papers is that we create a unified framework for inverse power control for cellular systems. The proposed algorithm links the existing algorithms to LMS type of adaptive algorithms. The modified filtered-x least mean square (MFxLMS) algorithm can be seen as a generalized adaptive inverse control method and several practical algorithms as special cases of it. In addition to theoretical analysis that is made more thoroughly in this paper than in our previous papers, we develop a practical quantized version of the algorithm and compare its performance to state-of-the-art algorithms. The proposed algorithm provides a fast adapting inverse power control solution that does not overshoot the power level as much after a fade as the conventional solution in [14]. Thus, it decreases interference to other users in these cases. We also propose an efficient way to implement the closed loop algorithm described in [15] as an enhanced version of the algorithm presented in [14].

Furthermore, we present novel fast simulation models for a fading channel and diversity. It was reported in [16] that Jakes' model [17] does not produce wide-sense stationary signals. The authors of [16] proposed to improve the model by randomizing the phase shifts of the low-frequency oscillators. We have modified Jakes' model further by randomizing also the frequency shifts in the model. Several simulation studies are performed with the practical power control algorithms both in additive white Gaussian noise (AWGN) and fading channels. The model is applicable to a multiple-input multiple-output orthogonal frequency division multiplexing (MIMO-OFDM) system with certain assumptions as discussed in Section 3. MIMO FxLMS algorithm has been recently studied for vibration control in [18].

The organization of the paper is as follows. Section 2 discusses related literature, and Section 3 presents the system model. Performance metrics are introduced in Section 4. The MFxLMS algorithm with the convergence analysis is presented with several other adaptive inverse power control schemes in Section 5. Achieved results are provided in Section 6, and conclusions with recommendations for further work are drawn in Section 7.

\section{Related literature}

Power control methods can in general be divided into water filling and channel inversion [6]. Basically, the difference between these two approaches is that the water filling allocates more power to the better channel instants whereas channel inversion aims at inverting the channel power gain while maintaining the desired signal strength at the receiver.
Several adaptive inverse control methods have been developed and studied in the literature for power control, e.g., [14, 15, 19-24]. The conventional 1-bit adaptive power control (CAPC-1) method [14, 19] employs delta modulation, i.e., adjusts the previous transmission power up or down by a fixed step. In this paper, the acronym CAPC- $x$ refers to conventional power control using $x$ bits in the power control command. Conventional inverse power control approaches have been proposed and used, e.g., for code division multiple access (CDMA), Third Generation Partnership Project (3GPP) long-term evolution (LTE), and TV white space transmission. A clear aim of these inverse methods is energy and interference reduction, to use only sufficient power to meet the transmission rate requirements. For example, CDMA power control employs both closed and open loop methods. In the open loop method, the mobile station measures the average received total power by an automatic gain control (AGC) circuit and adjusts its transmission power so that it is inversely proportional to the received power [25]. Nonlinear control is used to allow fast response to the reduced channel attenuation with a maximum of $10 \mathrm{~dB} / \mathrm{ms}$ but slow response to increased attenuation. This is to avoid additional interference to other users.

Required dynamic range with a limited feedback can be achieved by nonlinear quantization of feedback signaling [26] and variable step (VS) algorithms [27-30]. Nonlinear AGC control can be exponential or approximately exponential [3]. A simple way to compress power control commands is to operate the algorithm in decibel domain [20]. Logarithmic quantization such as $\mu$-law and $A$-law companding is used in speech codecs [26]. Companding amplifies weak input signals and compresses strong signals to save the needed number of bits to be transmitted. Companding is applied also for reducing peak-to-average power ratio (PAPR) in OFDM signals [31].

Many variable step size LMS algorithms have been proposed in order to improve the performance of the LMS algorithm by using large step sizes in the early stages of the adaptive process and small step sizes when the system approaches convergence [27-30]. The step size can be adapted, e.g., based on the received signal power [11] or the squared error signal $[19,20]$. Optimization of the step size has been studied in [32], where lag error of an adaptive system is also considered. This error is caused by the attempt of an adaptive system to track variations of the non-stationary input signal.

\section{System model}

The system model for adaptive transmission is illustrated in Fig. 1. The input data $x_{k}$ are binary phase-shift keying (BPSK) modulated and transmitted from the transmitter to the receiver over a fading channel. The algorithm can be used for any linear modulation scheme such as M-ary 


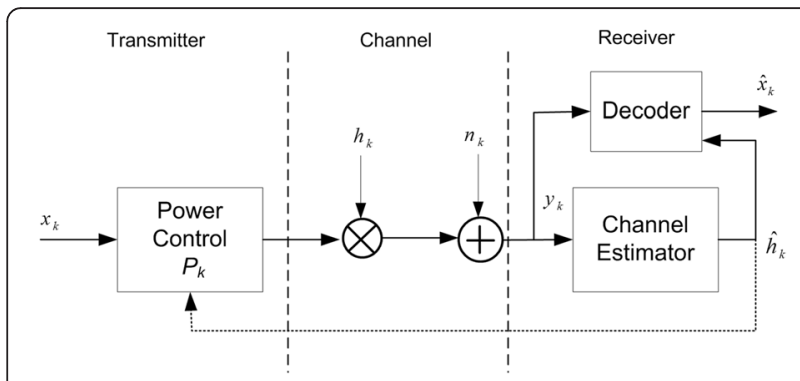

Fig. 1 System model for adaptive transmission

PSK and M-ary quadrature amplitude modulation (QAM) without any modifications. On the other hand, the analysis for each modulation scheme must be considered separately. The analysis for quadrature phaseshift keying (QPSK) is essentially the same since QPSK can be viewed as two independent BPSK signals in a frequency-nonselective channel. The received signal $y_{k}$ can be given as.

$$
y_{k}=x_{k} P_{k} h_{k}+n_{k}
$$

The complex gain of the channel is $h_{k}=\alpha_{k} e^{j \theta_{k}}$ and $n_{k}$ is the additive white Gaussian noise at time $k$. The amplitude of the fading gain is $\alpha_{k}$ and $\theta_{k}$ is the phase shift. The data are transmitted through the channel and the instantaneous transmit power $P_{k}$ is allocated based on the channel gain estimate $\hat{h_{k}}$ sent by the receiver. LMS estimation of the channel gain is done as.

$$
\hat{h_{k+1}}=\hat{h_{k}}+9 e_{k} x_{k}
$$

where $\vartheta$ is the step size of the algorithm and $e_{k}$ is the estimation error [1]. A typical value for the step size 9 is $0-0.99$. Larger values lead to faster convergence with the cost of reduced accuracy since noise averaging does not work so well [33].

We consider a slowly varying channel that can be modeled using the Doppler power spectrum [16, 17]. The rate of the channel variation, i.e., the effect of mobility, can be characterized by the Doppler frequency $f_{\mathrm{d}}$. We are using a flat Doppler power spectrum that corresponds to an urban environment where the transmitter is set above rooftop level [34].

\subsection{Sum-of-sinusoids fading channel}

To obtain a flat Doppler power spectrum, the timevariant channel gain of a channel with index $l$ is represented by the sum of complex exponentials as.

$$
h_{l}(k)=\frac{1}{\sqrt{N}} \sum_{i=1}^{N} e^{j\left(2 \pi f_{i, l} k+\phi_{i, l}\right)}
$$

where $N$ is the number of subpaths with the same delay, $f_{i, l}$ is the Doppler shift of the $i$ th subpath, ${ }_{i, l}$ is the random phase shift of the $i$ th subpath uniformly distributed in the range $[0,2 \pi]$, and $k$ is the time. The amplitudes of the subpaths in Eq. (3) are identical due to the flat spectrum. The average energy gain of the channel is normalized to unity [35]. The model is straightforward to generalize to multiple delays.

If the Doppler shifts of the complex exponentials are equally spaced in the interval $\left[-f_{\mathrm{d}}, f_{\mathrm{d}}\right]$, the channel gain (Eq. (3)) becomes periodic in time. Sampling in time domain corresponds to periodicity in frequency domain and vice versa [36]. Periodicity can be removed if the shifts are properly chosen. The Doppler spread is divided into $N$ equal size frequency bins. Within these bins, the frequencies $f_{i, l}$ differ a random uniformly distributed amount from the equal space solution. Thus, we obtain the whole Doppler spread to use in every simulation. The power spectrum is made symmetric with respect to zero frequency, which makes the autocorrelation function of the channel real. This selection also makes simulations faster. The random phases $\phi_{i, l}$ are not symmetric with respect to the zero frequency.

\subsection{Diversity channel}

A time-variant frequency selective channel model can be represented with a tapped delay line as.

$$
\beta(l, k)=\frac{1}{L} \sum_{l=1}^{L} h_{l}(k) \delta\left(\tau-\tau_{l}\right)
$$

where $L$ is the number of tap weights and $\tau_{l}$ is the delay of $l$ th tap generated using Eq. (3). Now, we have a flat impulse response instead of usual exponentially decreasing model. However, from power control point of view, this does not affect since the optimal demodulator for this signal is a coherent demodulator that collects the signal energy from all the received signal paths within the delay span 0 to $\tau_{L}$ [2]. In a diversity system, the transmitter power control algorithm should control the power of the diversity combiner output in the receiver. There is no loss in performance in dividing the total transmitted signal energy differently among the $L$ channels, and thus, the model does not change the comparison between the selected power control algorithms. Actually, the time-variant channel gain of the diversity channel can be given as.

$$
H(k)=\sqrt{\frac{1}{L} \sum_{l=1}^{L}\left|h_{l}(k)\right|^{2}}
$$

where $h_{l}(k)$ is the channel gain of the $l$ th diversity branch, generated using Eq. (3), and $L$ is the number of diversity branches. Equation (5) corresponds to the ideal maximal-ratio combining. The channel can thus represent also a frequency selective channel. From the 
subcarrier point of view, frequency selective channel looks frequency-nonselective in an OFDM system [2]. We assume that no intersymbol interference (ISI) or interpulse interference (IPI) is present since we use compressed pulses [2]. In general, a MIMO system with $M_{t}$ transmitter antennas and $N_{r}$ receiver antennas can be used as a diversity system having $M_{t} \times N_{r}$ independent diversity branches [37]. With OFDM system, those branches are made frequency-nonselective, and finally, by the use of orthogonal codes, the branches can be separated from each other. The channel must be slowly fading compared to the OFDM symbol rate so that intercarrier interference is avoided. The fading of the adjacent subcarriers is not uncorrelated, but this is typical in all OFDM systems, and it is in practice handled with frequency domain interleaving. Thus, with these assumptions, our system model represents also a MIMOOFDM system.

\section{Performance metrics}

Suitable performance metrics are needed to fairly compare the performance of the adaptive algorithms. One of the most important ones to consider is the signal-to-noise ratio (SNR) concept. The average transmitted and the average received energies are usually normalized by the receiver noise spectral density $N_{0}$ leading to the average transmitted SNR per symbol [35].

$$
\bar{\gamma}_{\mathrm{tx}}=\bar{E}_{\mathrm{tx}} / N_{0}
$$

and the average received SNR per symbol [35]

$$
\bar{\gamma}_{\mathrm{rx}}=\bar{E}_{\mathrm{rx}} / N_{0}
$$

The parameter $\bar{E}_{\mathrm{tx}}$ is the average transmitted energy per symbol, and $\bar{E}_{\mathrm{rx}}$ is the average received energy per symbol. Transmitted energy is a basic system resource. In a mobile terminal, it is taken from the battery of the transmitter and is therefore limited. Transmitted energy or equivalently transmitted SNR should be used as a performance metric in order to obtain fair comparisons between different adaptive transmission systems. In adaptive transmission, the transmitted signal is a function of the energy gain of the channel. The use of the received SNR as a performance criterion in adaptive transmission system studies can lead to misleading results as was shown in [35].

Learning curve, i.e., plotting the mean square error (MSE) against the number of iterations, can be used to measure the statistical performance of adaptive algorithms $[1,2]$. The MSE $J(k)$ can be approximated as.

$$
J(k)=\frac{1}{\eta} \sum_{i=0}^{\eta-1}\left|\varepsilon_{k-i}\right|^{2}
$$

where $\varepsilon_{k}$ is the error signal measured as a difference between the output of the adaptive algorithm and the desired signal. Parameter $\eta$ defines the number of samples used for averaging. Usually, MSE is compared to signal power, in this case transmission power.

\section{Adaptive power control methods}

\subsection{Theoretical inverse control methods}

If the truncated channel inversion (TCI) is used, the transmitted energy is [38].

$$
E_{\mathrm{tx}}(k)=\bar{E}_{\mathrm{tx}}\left(\sigma_{0} / \gamma_{\mathrm{H}}(k)\right)
$$

for $\gamma_{\mathrm{H}}(k) \geq \gamma_{0}$ and zero otherwise where $\sigma_{0}$ is a constant selected so that the average transmitted energy is $\bar{E}_{\mathrm{tx}}$. The quality of the channel is defined as $\gamma_{\mathrm{H}}(k)=$ $\bar{E}_{\mathrm{tx}}|H(k)|^{2} / N_{0}, \gamma_{0}, \gamma_{0}$ is a cutoff value, which is found by numerically maximizing (4.22) in [38], and $|H(k)|^{2}$ is the instantaneous energy gain of the channel. The cutoff value is $\gamma_{0}=0$ for full channel inversion. Channel inversion aims at maintaining the desired signal strength at the receiver by inverting the channel power gain based on the channel estimates.

\subsection{Adaptive FxLMS algorithm}

The power control structure based on the MFxLMS algorithm is introduced in Fig. 2. It approximates the channel inversion. In the following, we will present both original and modified versions of the algorithm. The FxLMS algorithm updates the coefficient $c_{k}$ of a one-tap filter as.

$$
c_{k}=c_{k-1}+w_{k}
$$

where $w_{k}=\mu x_{k}^{\prime} \varepsilon_{k}$ is the correction term, $\mu$ is the adaptation step size of the algorithm that regulates the speed and stability of adaptation, and $\varepsilon_{k}$ is the error signal to be minimized. The $c_{k}$ is the instantaneous transmission power at a time slot $k$. The filtered input signal for the FxLMS algorithm is $x_{k}^{\prime}=\left(\hat{x_{k}} \hat{h_{k}}\right)^{*}$, where $x^{*}$ is the complex conjugate version of $x, \hat{x}_{k}$ is the estimated input signal, and $\hat{h_{k}}$ is the estimated instantaneous channel gain. The filtered input signal is $x_{k}^{\prime}=\left|\hat{x_{k}} \hat{h_{k}}\right|$ for the MFxLMS algorithm, and the parameter $n_{k}$ is the additive white Gaussian noise. The channel can be modeled using Eqs. (3)-(5).

We have modified the conventional FxLMS structure by adding the absolute value blocks to the algorithm and having a wireless channel as a system to be controlled and inverted. In addition, a large difference in our system 


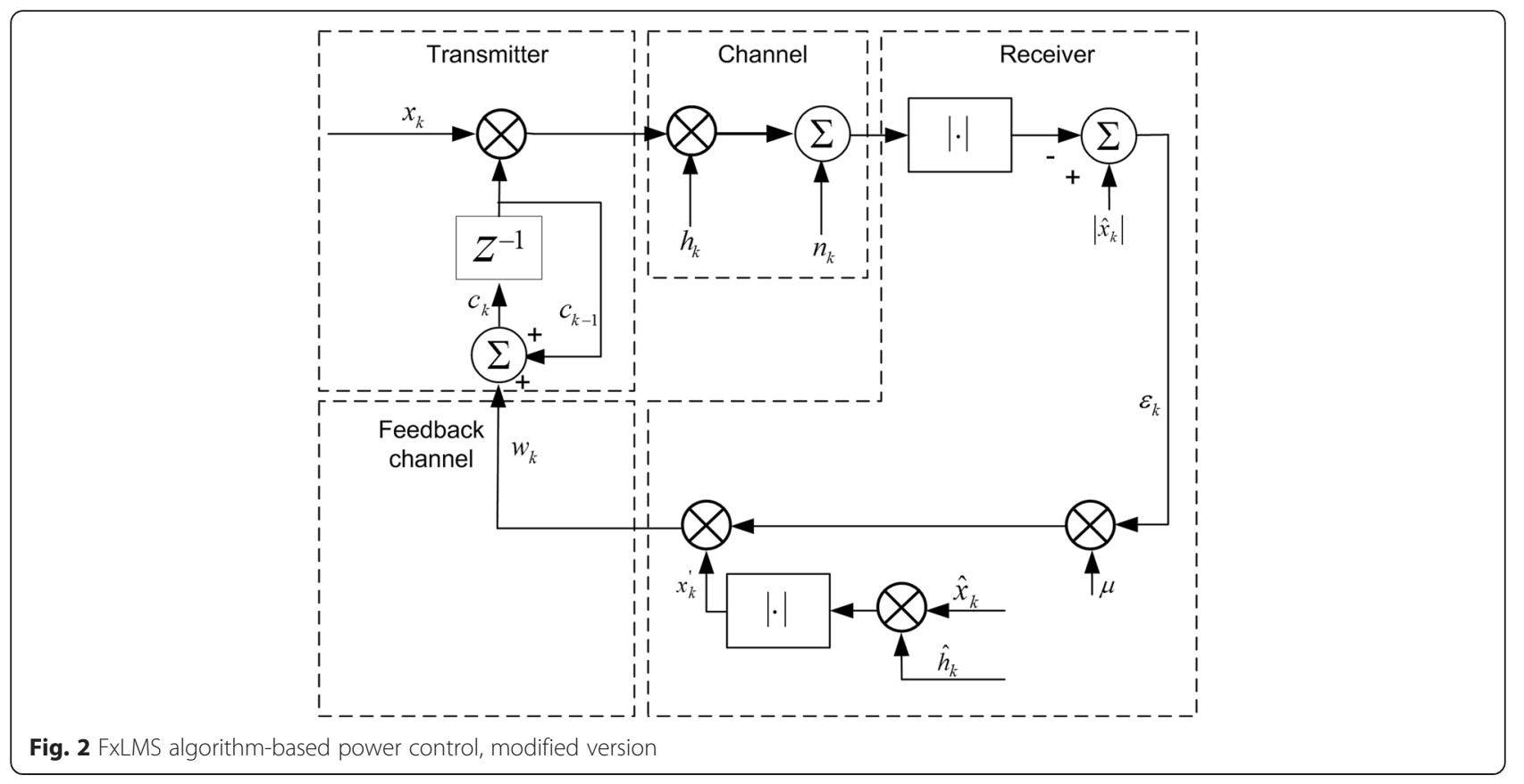

to conventional control systems comes with a separate receiver and transmitter. The main reason for the addition of absolute value blocks is that we are adjusting power levels and thus interested only in amplitude values, similar to AGC circuits [3]. Phases are not important from the power control point of view, and in this way, we can reduce control information to be carried. This also makes the system more robust since phases can change fast during deep fades and thus cause problems to the adaptive algorithm $[39,40]$.

The model is discretized using a matched filter [2], assuming slow changes compared to the symbol rate. Thus, we can use one sample of a symbol in the system model. We can reduce the complexity of the transmitter by doing the main part of the calculations at the receiver. This reduces also information in the feedback channel since only the correction term $w_{k}$ is sent to the transmitter. The filtered input signal $x_{k}^{\prime}$ affects the operation of the algorithm. Thus, the control structure is decision directed (DD) [2]. Error propagation is known in DD approaches and remedy strategies have been developed [41, 42]. It was proposed in [42] that pilot on request training (PRQT) is used to mitigate the error propagation. The pilot is requested when error propagation is detected in the system. We assume our system to operate with the PRQT principle. When the error probability is very small, we can assume $\hat{x_{k}}$ to be $x_{k}$.

\subsubsection{Convergence analysis for the MFxLMS power control algorithm}

The choice of initial conditions for the FxLMS algorithm is not critical [4]. The algorithm is stable if $\mu$ is small enough, and transients die out just as with the conventional LMS algorithm. A primary concern with the MFxLMS algorithm is its convergence to the optimal solution where $E\left[\varepsilon_{k}^{2}\right]$ is minimized. Since absolute value blocks make the analysis of the algorithm very complicated in a noisy channel, we will first analyze the conventional FxLMS algorithm that can also be used in power control. A general analysis for the FxLMS algorithm can be found in [43].

\section{(a) Coherent case}

Let us assume a time-invariant channel with perfect channel estimation, i.e., $\hat{h_{k}}=h$. The error signal is now

$$
\varepsilon_{k}=x_{k}-\left(h c_{k-1} x_{k}+n_{k}\right) \text {. }
$$

The control command (Eq. (10)) can be given as

$$
c_{k}=c_{k-1}+\left(x_{k} h\right)^{*} \mu \varepsilon_{k},
$$

and placing error signal (Eqs. (11) to (12)) yields

$$
c_{k}=c_{k-1}\left(1-\mu|h|^{2}\left|x_{k}\right|^{2}\right)+\mu h^{*}\left|x_{k}\right|^{2}-\mu h^{*} x_{k}^{*} n_{k} .
$$

Taking expected value of both sides leads to

$$
E\left[c_{k}\right]=\left(1-\mu|h|^{2} R\right) E\left[c_{k-1}\right]+\mu h^{*} R
$$

where $R=E\left[\left|x_{k}\right|^{2}\right]$. The white noise $n_{k}$ is assumed to be uncorrelated with the input $x_{k}$. In addition, we assume that the input $x_{k}$ is independent of the weight $c_{k}$ as in the analysis of the LMS algorithm in [4]. The first part 
of the function in the right-hand side will clearly form a geometric series that will converge only if the geometric ratio has a magnitude of less than unity,

$$
\left.|1-\mu| h\right|^{2} R \mid<1 .
$$

Therefore, we can define

$$
\begin{aligned}
E\left[c_{\infty}\right] & =\lim _{k \rightarrow \infty}\left(1-\mu|h|^{2} R\right)^{k} c_{0}+\frac{\mu R h^{*}}{1-\left(1-\mu|h|^{2} R\right)} \\
& =\frac{h^{*}}{|h|^{2}}=\frac{h^{*}}{h h^{*}}=\frac{1}{h} .
\end{aligned}
$$

Since the first part of the sum in Eq. (16) will approach zero, the algorithm converges exactly to the inverse of the channel gain. We can see from Eq. (15) that in order to keep the algorithm stable, the step size for updating the algorithm coefficients should be

$$
0<\mu<\frac{2}{R|h|^{2}}
$$

The optimal step size for the FxLMS algorithm lies in the middle of stability interval $[43,44]$. The convergence will be fastest with this selection. Thus, the optimal step size is now

$$
\mu_{\mathrm{opt}}=\frac{1}{R|h|^{2}}
$$

With this selection, the fixed step FxLMS algorithm is changed to the normalized version of it.

\section{(b)Non-coherent case}

From the power control point of view, we would only need the inverse of the absolute value of the channel gain instead of the result of Eq. (16) since we are interested in inverting the power level to maintain the received signal power at a constant level. Thus, let us now consider the MFxLMS algorithm with absolute value blocks in a timeinvariant, noiseless channel, assuming perfect channel estimation, i.e., $\hat{h_{k}}=h$. The error signal is given as

$$
\varepsilon_{k}=\left|x_{k}\right|-\left|h c_{k-1} x_{k}\right| \text {. }
$$

Thus, Eq. (10) becomes

$$
c_{k}=c_{k-1}+\mu|h|^{2}\left|x_{k}\right|^{2}-\mu\left|c_{k-1}\right|\left|x_{k}\right|^{2}|h| .
$$

In general, we should consider two separate cases: $c_{k}>$ 0 and $c_{k}<0$. However, there is no need to use negative values in power control since the solution we want to achieve is to maintain certain SNR at the receiver. The case $c_{k}<0$ leads to the converged solution that is a negative version of the solution for the case of $c_{k}>0$. When $c_{k}>0,\left|c_{k}\right|=c_{k}$. Now,

$$
c_{k}=\left(1-\mu\left|x_{k}\right|^{2}|h|^{2}\right) c_{k-1}+\mu\left|x_{k}\right|^{2}|h| .
$$

Convergence conditions for the MFxLMS algorithm can be found from this version quite straightforwardly, leading to the same solution as is shown in Eq. (15). Therefore, we can write

$$
c_{\infty}=\frac{1}{|h|}, \quad c_{k}>0 .
$$

The algorithm converges exactly to the inverse of the absolute value of the channel gain. We can see from the results above that in order to keep the algorithm stable, the step size for updating the algorithm coefficients should be exactly in the same interval as the one shown in Eq. (17). Thus, the optimal step size for the MFxLMS algorithm in a noiseless channel is given in Eq. (18).

Convergence of the MFxLMS algorithm when noise is present in the system becomes mathematically intractable due to the absolute value blocks. Now, Eq. (10) can be rewritten as

$$
c_{k}=c_{k-1}+\mu|h|\left|x_{k}\right|^{2}-\mu\left|h x_{k}\right|\left|h x_{k} c_{k-1}+n_{k}\right| .
$$

The algorithm cannot be analyzed straightforwardly due to absolute value of the term that includes noise. Simulations are also used instead of analysis in reference state-of-the art algorithm developments due to mathematical intractability $[14,15,19-21]$. Based on the simulations, the MFxLMS algorithm behaves and converges almost identically with the algorithm without absolute blocks in a fading channel when the transmitted SNR is high enough. Actually, the MFxLMS algorithm is more robust since fast phase changes do not affect its performance. Nonlinearity causes threshold phenomenon for the modified algorithm in low SNR regime that is always a problem in noncoherent systems using some combining or averaging.

\subsubsection{Time-variant channel}

Usually, the adaptation step size of the FxLMS algorithm is not time variant. The algorithm with a fixed adaptation step size corresponds to a first-order system. It cannot track the fastest changes in the time-variant channel without a lag error [32] that can be quite large. A better performance is achieved by optimizing the adaptation step size with the instantaneous power of the input signal. It means that the FxLMS algorithm with a fixed step size is changed to the normalized version of it. The normalized version of the FxLMS algorithm corresponds to the filtered-x recursive least squares (FxRLS) algorithm when $\mu=1-\lambda$ where $\lambda$ is the forgetting factor which gives exponentially less weight to older samples.

In a slowly fading channel, $h_{k}$ can be assumed to be constant over the memory [4] of the MFxLMS algorithm. 
Thus, the stability condition to the structure when noise is ignored and the channel state is known is the same as presented in Eq. (17) when $h$ is replaced by $h_{k}$. The optimal step size can be found for each different $h_{k}$ in Eq. (18) by replacing $h$ by $h_{k}$. Therefore, the optimal adaptation step size should be time variant. When the channel gain is estimated in Eq. (18), the system becomes unstable if this step size is used due to errors in the estimate [45]. To stabilize the control, the step size is given by.

$$
\mu_{\mathrm{s}}=\frac{a}{R\left|\hat{h_{k}}\right|^{2}+b}
$$

where $b$ is a small real number that prevents the adaptation step size to grow uncontrollably when the estimated received power is close to zero. Parameters $a$ and $b$ are dependent, e.g., on SNR and $L$, but the values need to be found experimentally to optimize the trade-off between lag error and noise averaging for different channel dynamics. Default values for these parameters can be given as $a=1 / \sqrt{L}$ and $b=0.2 / \bar{\gamma}_{\mathrm{rx}}$ where $\bar{\gamma}_{\mathrm{rx}}$ is the received SNR defined in Eq. (7). Smaller $a$ means slower convergence, better noise averaging, and a larger lag error while the parameter $b$ has an opposite effect.

\subsubsection{Quantized MFxLMS power control}

In the following sections, only the MFxLMS algorithm is considered. In practice, the power control command has to be quantized while obtaining a decent performance. In the case of the MFxLMS algorithm, the signal $w_{k}$ has to be fed back to the transmitter as shown in Fig. 2. It is good to quantize frequently occurring small values of the signal in more detail and then use coarser steps for the less frequent large signal levels to preserve needed information [26]. The signal $w_{k}$ is first compressed, then quantized uniformly, and sent to the channel. The received signal is expanded to get close to the original version of the power control command.

The $\mu$-law compression is defined for real input signal $w_{k}$ as

$$
v_{k}=F\left(w_{k}\right)=\operatorname{sgn}\left(w_{k}\right) \frac{V \ln \left(1+\mu_{\mathrm{q}}\left|w_{k}\right| / V\right)}{\ln \left(1+\mu_{\mathrm{q}}\right)} .
$$

where $V$ is the peak magnitude of the input signal. This is also a peak value of the output. A typical value for the compression parameter $\mu_{\mathrm{q}}$ is between 50 and 300. In our case, we have quantized the signal in the range $[-1,1]$ to be able to effectively combat the deep fades even though the average power of the signal $v_{k}$ is roughly 0.1 . The maximum value is close to unity during the deepest fades. We have not scaled the signal before quantization. If the signal is scaled up, the clipping is increased while the quantization noise is reduced. Scaling down reduces clipping but increases noise. Received quantized signal $q_{k}$ is expanded using

$$
\begin{aligned}
\hat{w_{k}}= & F^{-1}\left(q_{k}\right) \\
= & \operatorname{sgn}\left(q_{k}\right)\left(\frac{V}{\mu_{\mathrm{q}}}\right) \\
& \times\left(e^{\left|q_{k}\right| \ln \left(1+\mu_{\mathrm{q}}\right) / V}-1\right), \quad 0 \leq\left|q_{k}\right| \leq 1 .
\end{aligned}
$$

The proposed practical version of our MFxLMS algorithm allows fair comparison with other practical algorithms presented in the literature.

\subsection{Conventional adaptive power control}

Typically, the time interval between power control commands in CDMA systems is around $1 \mathrm{~ms}$ [14]. The method is shown in Fig. 3. The base station measures the average received power over $m$ symbols and compares it to a reference signal to interference plus noise (SINR) level $\gamma_{\text {ref. }}$ As a result of a comparison, the base station tells the mobile station to adjust its transmission power upwards when the error signal $\delta_{k}$ is positive or downwards with negative error by a control step size $\Delta P$. Practical CAPC-1 method $[14,19]$ uses $1 \mathrm{~dB}$ steps. The power control algorithm can be written as.

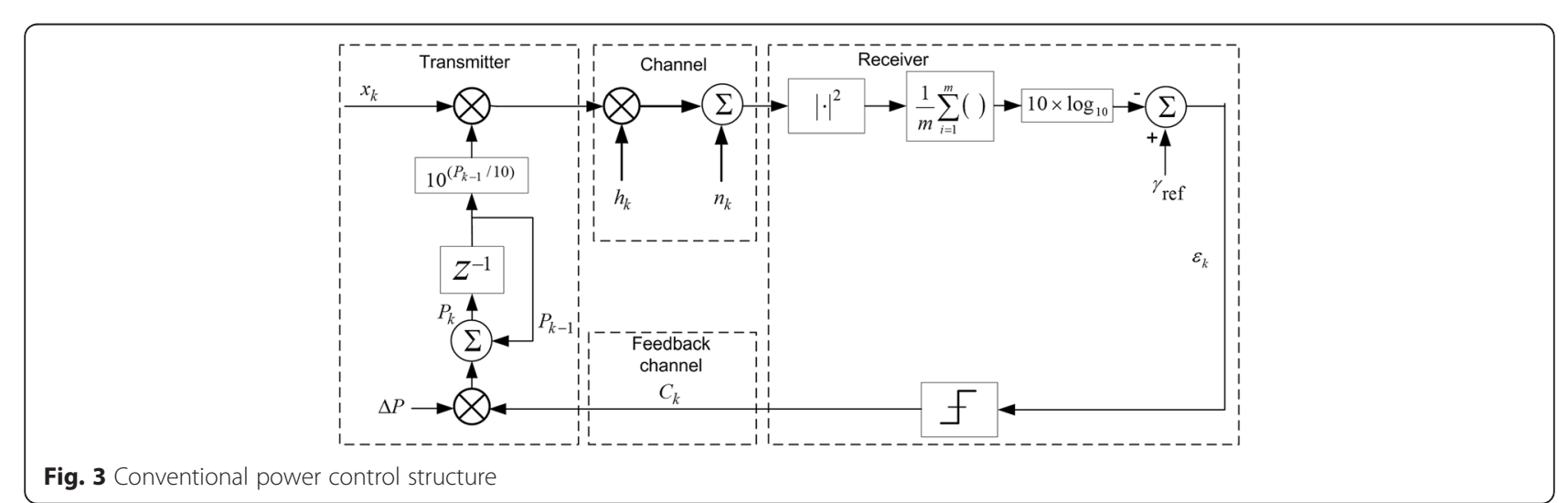




$$
P_{k}=P_{k-1}+C_{k} \Delta P \quad[d B]
$$

where the power control command is

$$
C_{k}=\left\{\begin{array}{cc}
+1, & \delta_{k}>0 \\
-1, & \delta_{k} \leq 0
\end{array}\right.
$$

The weakness of this fixed-step power control method is that it is too slow to track changes in a fading channel.

\subsection{Variable step adjustment power control}

Variable step power control methods have been proposed to overcome the weakness of the fixed-step solution. The basic idea is that when the power of received signal is far from the desired, the control step is increased to reach the desired level faster. A recently proposed 2-bit version of the CAPC (CAPC-2) method is described in [15] where power control command $C_{k}$ values are $C_{k}=\{-4,-1,1,4\}(\mathrm{dB})$. In the mentioned document [15], only step sizes are given. No rules how to use them in practice are included. Based on the simulation studies, we have conducted the following rule that was found to achieve a good performance:

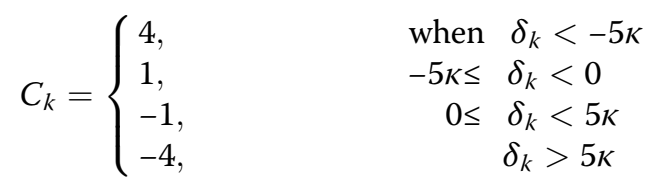

where $\kappa=0.5 \Delta P$. An asymmetric 3-bit version of the conventional adaptive power control (CAPC-3) proposed in [20] is

$$
C_{k}=\left\{\begin{array}{lcl}
3, & \text { when } \quad \delta_{k}<-5 \kappa \\
2, & -5 \kappa \leq & \delta_{k}<-3 k \\
1, & -3 \kappa \leq \delta_{k}<-k \\
0, & -\kappa \leq \delta_{k}<\kappa \\
-1, & \kappa \leq \delta_{k}<3 \kappa \\
-2 & \delta_{k}<3 \kappa
\end{array}\right.
$$

Variable step algorithms can be implemented with the structure depicted in Fig. 3. The only difference to the 1-bit CAPC method is in the quantization, i.e., more bits are used for power control commands in CAPC-2 and CAPC-3.

\subsection{Comparison between the FxLMS and conventional algorithms}

The idea to use the FxLMS algorithm started from the observation that analogy can be seen between the control structure in Fig. 3 and the LMS algorithm. Actually, the conventional algorithms can be seen as a special case of the FxLMS algorithm. The following modifications are needed from the MFxLMS structure in Fig. 2 to the CAPC structure in Fig. 3: (1) First, the CAPC structure uses square-law detection instead of envelope detection used in the MFxLMS structure. These have shown to provide comparable performance but the former is usually easier to analyze [46] while the latter allows a larger dynamic range [47]. (2) The CAPC method uses averaging to remove noise. The LMS algorithms are in principle based on exponential averaging [1]. An additional averaging block could be used as well, but it does not provide additional performance gain for the algorithm [48]. It is better to use instantaneous gradient estimates as is used in our power control structure. (3) Companding, i.e., going first to decibel domain (compressing) and then back to linear domain (expanding), is used in the CAPC algorithm. Compressing is used to cover the large dynamic range in a fading channel. In the practical MFxLMS structure, companding focuses on the task of nonlinear quantization, i.e., to reduce the number of bits in the power control command. (4) Onebit quantization is used in the CAPC- 1 method to simplify feedback signaling. The MFxLMS method is using quantization as in Eqs. (25)-(26). Power control command needs to be more than 1 bit for variable step power control. That is true also for the variable step algorithms that are based on the structure shown in Fig. 3. (5) The CAPC-1 method uses a fixed scaling factor $\Delta P$ whereas in the MFxLMS method, the step size scales based on the channel state. The similarities between the CAPC methods and the LMS method are so clear that the MFxLMS method can be seen as a generalization of inverse power control approaches.

\section{Results}

\subsection{Power control over an AWGN channel}

We performed simulations for FxLMS variants with a fixed channel gain $h=1$. The error signal $\varepsilon_{k}$ used in the MSE calculations is given in Eq. (11) with $c_{0}=0$, leading to $\varepsilon_{0}=1$ that is set as the first value to Eq. (8). The parameter $\eta$ used in the simulations was $\eta=6$, increasing from $\eta=1$ in the beginning until enough samples for $\eta$ = 6 were achieved. Ensemble averaging over 100 independent trials was performed to obtain the results for the FxLMS and the MFxLMS algorithms. We used BPSK signal in transmission and thus the choice of $\mu=1$ corresponds to the normalized algorithm using the optimum step size defined in Eq. (18). As expected, the larger the step size is the higher the converged mean squared error is. The performance of the algorithms is almost identical in the AWGN channel.

Learning curves for the practical algorithms are shown in Fig. 4 together with the normalized MFxLMS algorithm and the normalized FxLMS algorithm, using $20 \mathrm{~dB}$ received SNR. Numbers of iterations to the convergence are $28,10,5$, and 4 for the CAPC-1, CAPC-3, CAPC-2, and MFxLMS algorithms, respectively. Used 


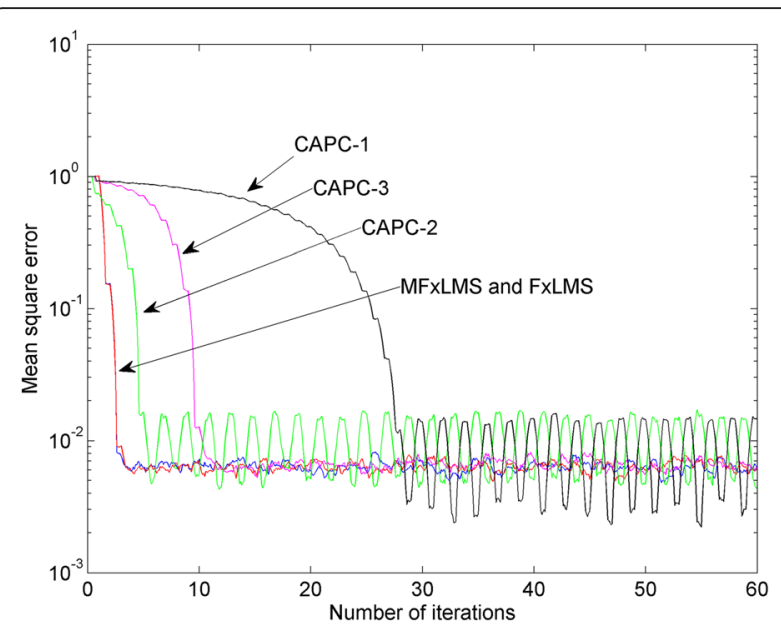

Fig. 4 Learning curves for studied algorithms in an AWGN channel

error signal in simulations for CAPC- $x$ algorithms was $e_{k}=x_{k}-\left(P_{k-1} h_{k}+n_{k}\right)$ to obtain a fair comparison with the MFxLMS results. The step sizes for CAPC- $x$ algorithms are defined in Eqs. (28)-(30) and the parameter $\gamma_{\mathrm{ref}}=20 d B$. For the criterion for the convergence, we used $10 \%$ misadjustment [4].

The CAPC- 1 is the slowest one due to fixed step size and the variable step size algorithms clearly outperform it. The CAPC-2 is faster than CAPC-3 since it uses a larger maximum step size for fast adaptation. CAPC-1 and CAPC-2 algorithms adapt the power up and down all the time. Other variable step algorithms can set the power to the wanted level and keep it there.

\subsection{Power control over a fading channel with the non- quantized MFXLMS algorithm}

Both conventional and modified versions of the FxLMS algorithm operate well in the AWGN channel as expected.
However, robustness of the FxLMS is not as good as the robustness of the MFxLMS algorithm when we look at the performance in the fading channel modeled with Eq. (3). Two different channel realizations are considered in Fig. 5. It shows the received SNR levels for the simulations over a fading channel modeled using Eq. (3) with value of $N=12$ and $f_{\mathrm{d}}=10 \mathrm{~Hz}$. The LMS channel estimation with parameter value of $\vartheta=0.1$ is used in the FxLMS simulations. In addition, the values of $a=1$ and $b=0.2 / \bar{\gamma}_{r x}$, where $\bar{\gamma}_{\mathrm{rx}}$ is the received SNR, were chosen for Eq. (24). The larger SNR is used, the more stable the control is and the smaller correction term is needed. In all the shown simulations, the power control update rate was $1000 \mathrm{~Hz}$.

In the first row in Fig. 5, the channel variations both in phases and amplitudes are not too fast for the algorithms to make inversion accurately. The performance of the conventional and modified FxLMS algorithms is almost identical. However, in the second row, the faster phase variations during deep fades [39] clearly cause problems to the conventional FxLMS algorithm.

The MFxLMS algorithm performs robustly, and the received SNR variation remains at an acceptable level since it follows the amplitude variations rather well also during the deep fades. Fig. 6 presents performance of the FxLMS and the MFxLMS algorithms in a fading channel in low and medium SNR regimes when the channel realization of row 1 of the Fig. 5 is used. When the transmitted SNR is above $8 \mathrm{~dB}$, the performance of the algorithms measured with standard deviation of received SNR is almost identical. The more robust MFxLMS obtains better performance than the conventional FxLMS below $8 \mathrm{~dB}$ due to problems caused by rapid phase variations to the latter. However, the performance of the MFxLMS collapses when the transmitted SNR drops below $5 \mathrm{~dB}$ while the FxLMS operates also below this limit. The reason for the collapse is the
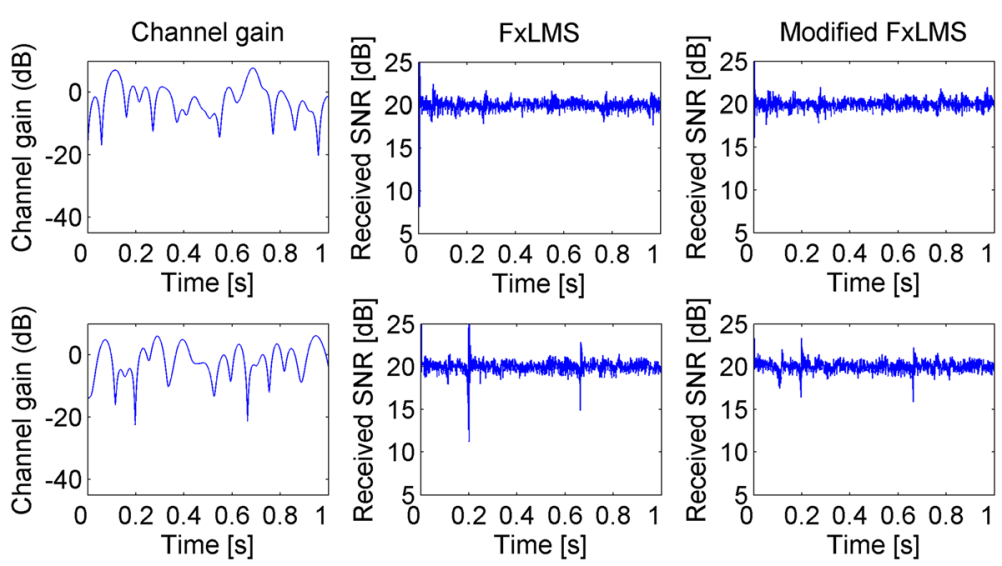

Fig. 5 Performance of the FxLMS algorithm in two different fading channels 


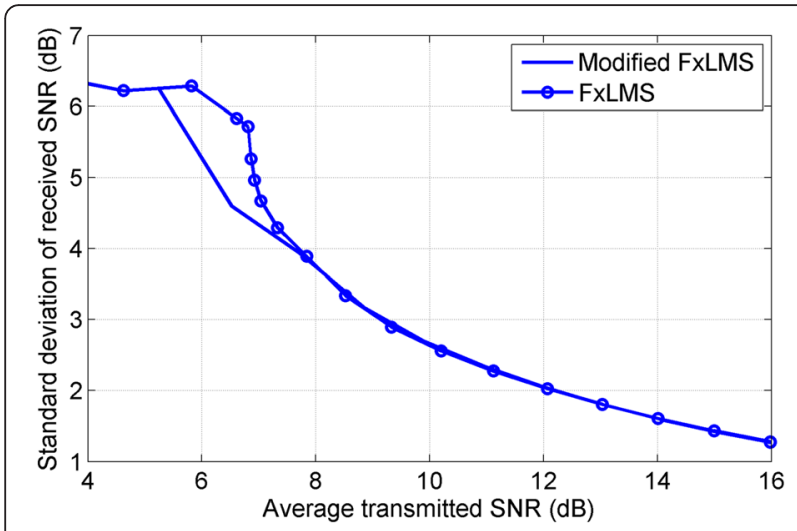

Fig. 6 Performance of the FxLMS algorithm in low and medium SNR regimes inclusion of noise term in Eq. (23) inside the last absolute value term. When the noise term is strong enough compared to the signal power, the algorithm cannot converge anymore. Above this performance limit, the MFxLMS is more robust and provides either equal or better performance compared to the conventional FxLMS. Thus, in all the remaining results, only the MFxLMS algorithm is considered in comparison with the CAPC- $x$ algorithms.

Results with the channel model shown in the row two of the Fig. 5 are presented in Fig. 7. With the CAPC-1 method, the received SNR is too low during a deep fade. Then, the transmission power is adjusted upwards, and because of lag error, the power is too high for a while. The variable step methods perform better. The CAPC-3 and MFxLMS methods can keep the received signal close to the desired value. The CAPC-2 and CAPC-1
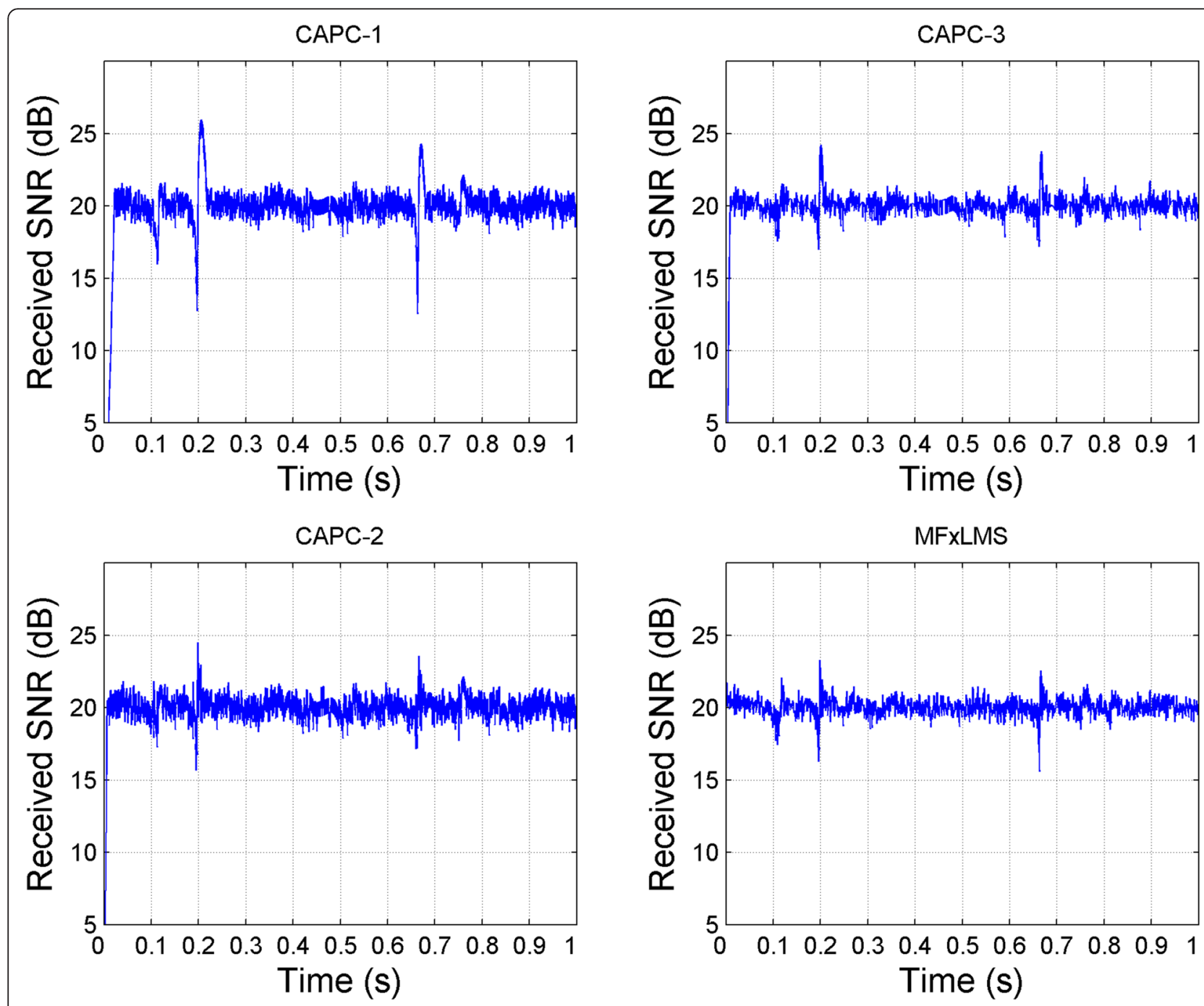

Fig. 7 Received SNR values with different power control methods 
methods are changing the power by $1 \mathrm{~dB}$ up and down even when they are close to the target level.

Variable step size methods have larger step sizes which make adaptation faster. This can be seen in the rise times in Table 1. The rise time is the time required for the received signal to change from the initial value, when transmitted signal is $0 \mathrm{~dB}$, to the required $20 \mathrm{~dB}$ value in a time-variant channel (Eq. (3)) using the same parameters as for generating the Fig. 5. The results are average values over several simulations. The MFxLMS algorithm sets the transmission power to the required level fast. In addition, the system is able to compensate deep fades without suffering lag error. That is the reason for the better performance for adaptive step size algorithms.

It is impossible to obtain identical received and transmitted SNRs since different algorithms use different amount of transmitted energy for communication due to their different adjustment methods. However, the difference is very small between CAPC-2, CAPC-3, and MFxLMS methods as shown in Table 1 . Thus, the performance comparison between these methods is fair. The performance of the CAPC-1 method is decreased since it is spending more time during deep fades with a lower power and consequently the outage time is also higher. Standard deviation of the received SNR, averaged in the decibel domain, shows clearly the gain of using adaptive step sizes in control with the studied control command rate. Standard deviation is measured after the rise time to exclude large differences between the required SNR and the actual signal level at that time. The MFxLMS algorithm achieves the best performance among the compared algorithms.

The bit error rate (BER) performance of the studied algorithms in the channel (Eq. (5)) when $L=1$ is considered in Fig. 8. The same metric was applied in [49] to compare fixed step and adaptive step power control. Simulations are carried out to establish the effect of power control step size (variable versus fixed) on the average BER performance. BPSK modulation is used in the simulations and its BER performance in AWGN channel plotted as a reference. The performance of the full channel inversion (FCI), referring to Eq. (9) with $\gamma_{0}=$ 0 , and the optimal TCI in a known channel are plotted as references to show the effect of adaptation in the BER performance. The difference of roughly $5 \mathrm{~dB}$ between the FCI

Table 1 Performance of the practical algorithms

\begin{tabular}{llcc}
\hline & $\begin{array}{l}\text { Rise } \\
\text { time }(\mathrm{ms})\end{array}$ & $\begin{array}{l}\text { Standard } \\
\text { deviation }(\mathrm{dB})\end{array}$ & $\begin{array}{l}\text { Average transmitted } \\
\text { SNR (dB) }\end{array}$ \\
\hline CAPC-1 & 19 & 1.186 & 25.46 \\
CAPC-2 & 7 & 0.798 & 26.25 \\
CAPC-3 & 9 & 0.613 & 26.31 \\
MFXLMS & 4 & 0.573 & 26.18 \\
\hline
\end{tabular}

and AWGN curves is caused by fading. The difference can be reduced with diversity. In the low SNR regime, the noise error is the dominating source of errors and the variable step algorithms are not performing better than the CAPC-1 method that was studied with two different step sizes, $\Delta P=0.5 \mathrm{~dB}$ and $\Delta P=1 \mathrm{~dB}$. The crossing in the BER curves between the MFxLMS and CAPC-1 methods around $12 \mathrm{~dB}$ SNR is due to effect of noise. When the SNR is higher, the standard deviation of the MFxLMS and the corresponding BER values are smaller. Variable step methods are using larger step sizes to correct the errors caused by the noise and that makes their performance worse in the low SNR regime. Smaller step sizes are better for noise averaging.

Also, the theoretical FCI method is worse than the CAPC-1 method in the low SNR regime since it allocates more power to the deep fades whereas the CAPC1 method cannot invert the channel totally, making it actually a truncated algorithm. When the SNR is increasing, the variable step methods can follow better the channel fading. The CAPC-1 method is too slow to invert the channel during fast changes especially with the smaller step size and the lag error makes the performance of it worse when SNR is increasing. The FxLMS method outperforms the other algorithms in the high SNR regime when the fastest converging step size defined in Eq. (24) is used, i.e., with $a=1$. However, during low SNR values, the smaller step size is better due to better noise averaging properties. The FCI performance approaches the TCI curve when SNR increases since the probability of outage of the TCI method is reducing.

\subsection{Power control over a diversity channel with the quantized MFxLMS algorithm}

The previous results are provided for the non-quantized MFxLMS algorithm to see its capabilities. Quantized version is needed to verify the practicality of the algorithm. The experiments were made over the diversity channel since that would be an obvious feature to be used in practical systems. The diversity channel with $L=$ 2 branches for simulation studies was generated using Eq. (5) and parameter values $N=12$ and $f_{\mathrm{d}}=10 \mathrm{~Hz}$. In order to see the effect of companding in the results, we made several simulation runs where we used either companding or pure quantization the feedback channel. The standard deviation of the received SNR of the algorithm with quantization was significantly lower with companding. Thus, we use companding in the following simulations. In addition to the proposed use of sending the correction term (Eq. (10)) in the feedback channel, we made experiments by sending the signal $c_{k-1}$ in the feedback channel to minimize calculations at the transmitter. However, the signal level variation using the correction term $w_{k}$ is smaller in the diversity channel, providing better performance with 


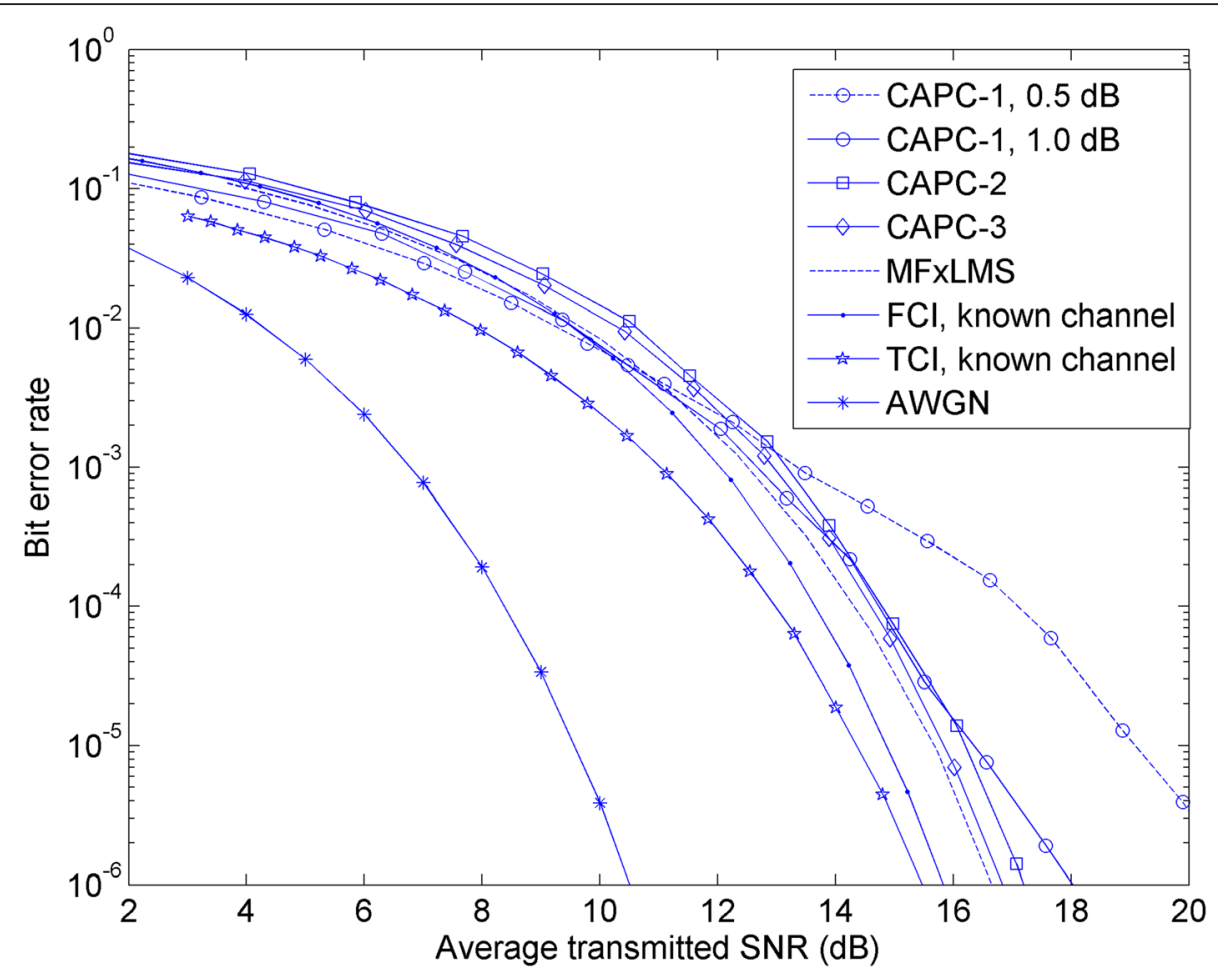

Fig. 8 The bit error rate as a function of average transmitted SNR in channel (Eq. (5)), $L=1$

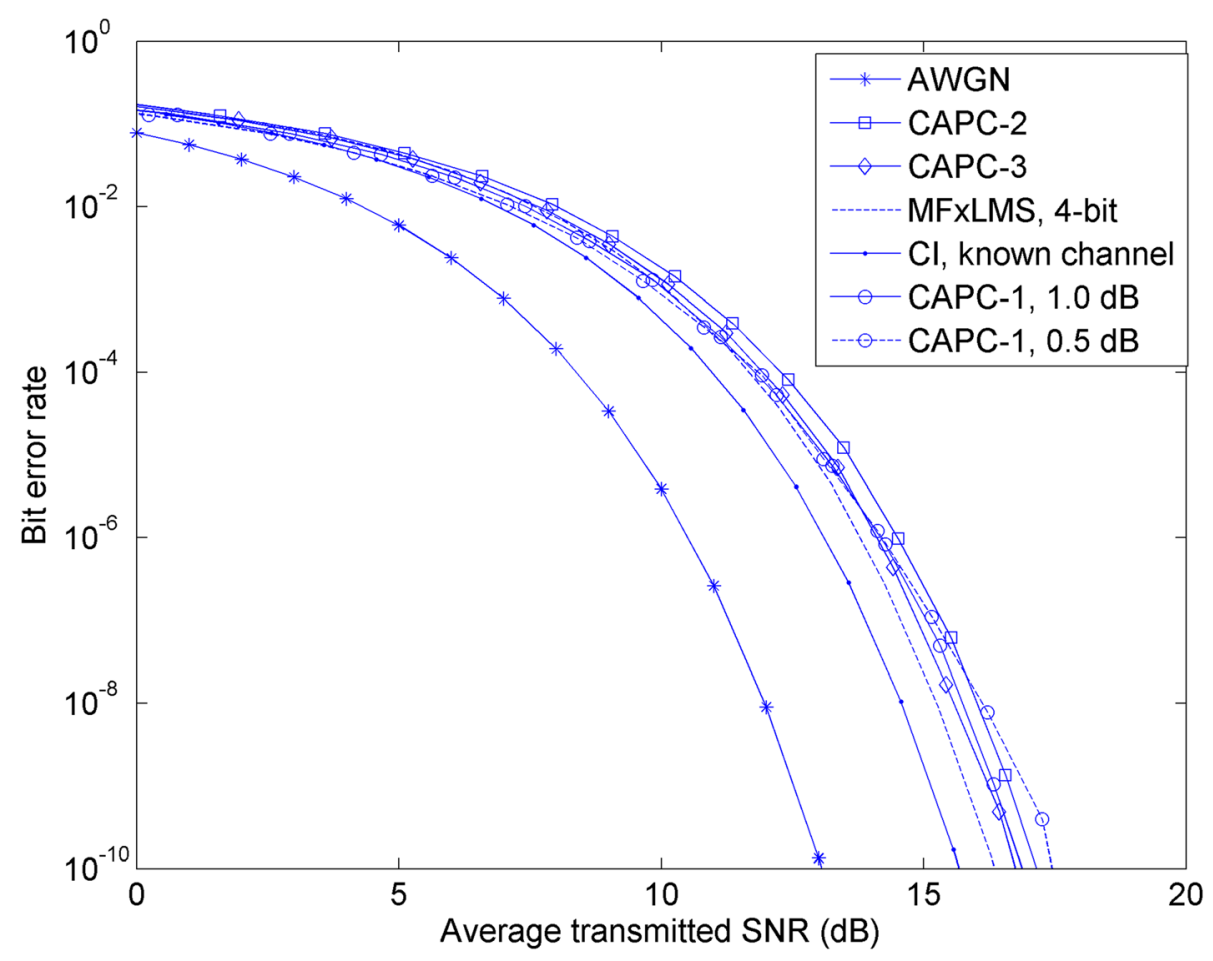

Fig. 9 Bit error rate performance comparison in a diversity channel $(L=2)$ 
the quantization. Simulation results using the quantized correction term are shown in Figs. 9 and 10.

The bit error rate performances of the studied algorithms are shown in Fig. 9 as a function of transmitted SNR. The performance of the full channel inversion in a known channel is plotted as a reference. It is actually a better reference in a diversity channel than in a channel without diversity where truncation gives a clear advantage. Full channel inversion without cutoff is the optimal inversion method in a diversity channel [12]. Control rate of the adaptive algorithms is $1000 \mathrm{~Hz}$. Control step size of the MFxLMS algorithm in a diversity channel was experimentally found to provide good tradeoff between lag error and noise averaging when the parameters in Eq. (24) were $a=1 / \sqrt{L}$ and $b=0.2 / \bar{\gamma}_{\mathrm{tx}}$. With higher SNRs, the inversion is more accurate due to reducing effect of the noise error. All the tested algorithms work rather well in a diversity channel. The MFxLMS algorithm needs less SNR than other adaptive algorithms to achieve BER $<10^{-4}$ due to accuracy of the adaptation. Very close to the performance of the non-quantized MFxLMS algorithm is achieved with a 4-bit power control command. The performance of the MFxLMS algorithm approaches the ideal inversion when the channel is changing more slowly. The performance differences between the algorithms in the high SNR regime can be well understood when we look at the accuracy of the algorithms measured with the standard deviation of received SNRs.

It can be seen from the results shown in Fig. 10 that the MFxLMS control achieves comparable performance to the best earlier algorithm studied, i.e., the CAPC-3 method. Accuracy of the CAPC-1 and CAPC-2 algorithms is restricted due to the minimum step size of $1 \mathrm{~dB}$. The same crossing as detected in the BER curves between the MFxLMS and CAPC-1 methods around $12 \mathrm{~dB}$ SNR is

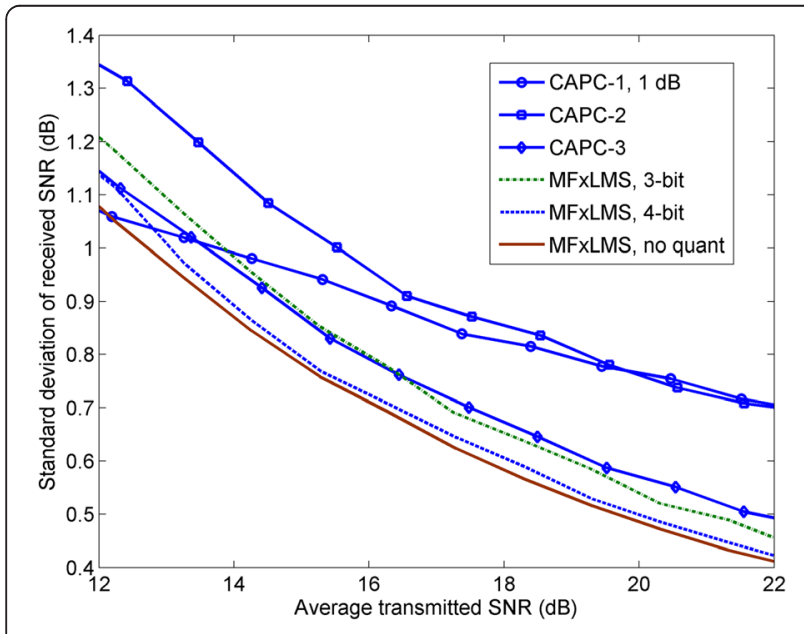

Fig. 10 Performance comparison in a diversity channel $(L=2)$ seen also in Fig. 10. When the SNR is higher, the standard deviation of the MFxLMS and the corresponding BER values are smaller. In a diversity channel, the additional larger step size of the CAPC-2 decreases the accuracy of the control compared to the simple CAPC- 1 control since the fading can be controlled with smaller steps. Variable step size algorithms are still outperforming fixed step algorithms in a diversity channel in the high SNR regime. However, the gain is achieved by using a higher feedback channel rate.

In order to see the effect of control rate to the accuracy of the control, simulations were performed with two different control rates, 1000 and $500 \mathrm{~Hz}$. Results are shown in Fig. 11. Main comparison is made between the most accurate variable step algorithms, the MFxLMS and the CAPC-3 algorithm. CAPC-1 results with $1000 \mathrm{~Hz}$ control rate using $1 \mathrm{~dB}$ step size are provided as a reference curve. The results show that reduction in the control rate still keeps the accuracy of variable step algorithms better than with a 1-bit algorithm with $1000 \mathrm{~Hz}$ rate. The number of control bits needed to send over the feedback channel is 1500 and $2000 \mathrm{bits} / \mathrm{s}$ for variable step CAPC and MFxLMS algorithms, respectively. CAPC-1 with a higher control rate requires only $1000 \mathrm{bits} / \mathrm{s}$, i.e., with a proper step size selection it gives rather good performance with a low feedback control rate. The rate depends on the fading rate of the channel and can be decreased, e.g., when higher order diversity is applied.

\section{Conclusions}

We have developed the MFxLMS algorithm for inverse power control. We analyzed the algorithm in a noiseless channel, and simulations show that it converges well also in a noisy channel. The proposed algorithm provides a unified framework for many existing practical algorithms

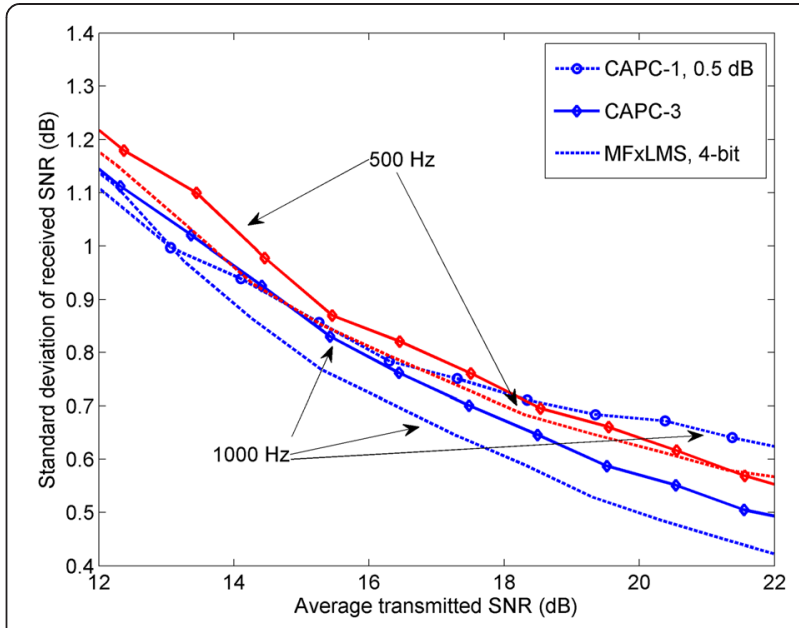

Fig. 11 Performance comparison in a diversity channel $(L=2)$ with different control rates 
and can be seen as a generalization of inverse power control algorithms. We compared the proposed method to the well-known CAPC-1 method and its variable step variants. Simulations in fading channels with diversity show that the best conventional algorithms give comparable performance to the theory-based MFxLMS solution. However, the most important contribution is the linking of the LMS-based theory and conventional power control algorithms that have traditionally been developed in separate paths.

An interesting future topic would be to study the optimization of the step size (Eq. (24)) of the MFxLMS algorithm in a time-variant channel. Some related work has been done for direct estimation and decision feedback equalization in [32], but more investigation is needed to find solutions for inverse control. Another interesting problem to study would be the development of the algorithm to handle vector type signals. The algorithm could be able to take into account the correlation between subcarriers in the OFDM system.

\section{Competing interests}

The authors declare that they have no competing interests.

\section{Acknowledgements}

This work was performed in the framework of the SANTA CLOUDS and SOCRATE projects, partly funded by the Finnish Funding Agency of Technology and Innovation. In addition, the work has been funded by VIT's own AWARENESS project. Authors would like to thank Prof. Simon Haykin for his encouraging comments that helped in preparing the manuscript.

Received: 29 September 2015 Accepted: 27 January 2016

Published online: 05 February 2016

\section{References}

1. B Widrow, SD Stearns, Adaptive signal processing (Prentice-Hall, New Jersey, 1985)

2. JG Proakis, Digital communications, 4th edn. (McGraw-Hill, New York, 2001)

3. H Meyr, G Ascheid, Synchronization in digital communications (Wiley, New York, 1990)

4. B Widrow, E Walach, Adaptive inverse control (Prentice-Hall, New Jersey, 1996)

5. MT Akhtar and W Mitsuhashi, A modified normalized FxLMS algorithm for active control of impulsive noise, in European Signal Processing Conference (EUSIPCO), Aalborg, Denmark, August 2010

6. E Biglieri, J Proakis, S Shamai, Fading channels: information-theoretic and communication aspects. IEEE Trans. Inf. Theory 44(6), 2619-2692 (1998)

7. TD Novlan, HS Dhillon, JG Andrews, Analytical modeling of uplink cellular networks. IEEE Trans. Wirel. Commun. 12(6), 2669-2679 (2013)

8. DR Morgan, An analysis of multiple correlation cancellation loops with a filter in the auxiliary path. IEEE Trans. Acoust. Speech Signal Process. ASSP28(4), 454-467 (1980)

9. JC Burgess, Active adaptive sound control in a duct: a computer simulation. J. Acoust. Soc. Am. 70(3), 715-726 (1981)

10. B Widrow, D Shur, and S Shaffer, On adaptive inverse control, in 15th Asilomar Conference on Circuits, Systems and Computers (AsilomarSSC), pp. 185-189, November 1981

11. M Höyhtyä, A Mämmelä, Adaptive inverse power control using an FxLMS algorithm, in IEEE 65th Vehicular Techonology Conference (VTC Spring), 2007, pp. 3021-3025

12. M Höyhtyä, A Kotelba, A Mämmelä, Practical adaptive transmission with respect to rational decision theory, in IEEE 69th Vehicular Techonology Conference (VTC Spring), 2011

13. M Höyhtyä, A Sahai, D Cabric, A Mämmelä, Energy efficient inverse power control for a cognitive radio link, in Second International Conference on Cognitive Radio Oriented Wireless Networks and Communications (CrownCom), 2007, pp. 264-271
14. A Salmasi, KS Gilhousen, On the system design aspects of code division multiple access (CDMA) applied to digital cellular and personal communication networks, in IEEE 41st Vehicular Techonology Conference (VTC), 1991, pp. 57-62

15. E-UTRA; Physical layer procedures, 3GPP, TS 36.213 (V10.4.0), 2011.

16. MF Pop, NC Beaulieu, Limitations of sum-of-sinusoids fading channel simulators. IEEE Trans. Comm. 49(4), 699-708 (2001)

17. WC Jakes, Microwave mobile communications (Wiley, New York, 1974)

18. Q Huang, X Zhu, Z Gao, S Gao, E Jiang, Analysis and implementation of improved multi-input multi output filtered-X least mean square algorithm for active structural vibration control. Struct. Control Hlth. 20(11), 1351-1365 (2013)

19. K.S. Gilhousen, R. Padovani, and C. E. Wheatley, U.S. Patent 5056 109, 8 October 1991

20. Y-J Yang, J-F Chang, A strength-and-SIR-combined adaptive power control for CDMA mobile radio channels. IEEE Trans. Veh. Technol. 48(6), 1996-2004 (1999)

21. T Frantti, Multiphase transfer of control signal for adaptive power control in CDMA systems. Control Eng. Pract. 14(5), 489-501 (2006)

22. C-Y Yang, B-S Chen, Robust power control of CDMA cellular radio systems with time-varying delays. Signal Process. 90(1), 363-372 (2010)

23. W Cheng, X Zhang, $H$ Zhang, QoS-aware power allocations for maximizing effective capacity over virtual-MIMO wireless networks. IEEE J. Sel. Areas Commun. 31(10), 2043-2057 (2013)

24. H ElSawy, E Hossain, On stochastic geometry modeling of cellular uplink transmission with truncated channel inversion power control. IEEE Trans. Wirel. Commun. 13(8), 4454-4469 (2013)

25. EG Tiedemann, AB Salmasi, KS Gilhousen, The design and development of a code division multiple access (CDMA) system for cellular and personal communications, in IEEE First International Symposium on Personal, Indoor and Mobile Radio Communications (PIMRC), 1991, pp. 131-136

26. LR Rabiner, RW Schafer, Introduction to digital speech processing. Foundations and Trends in Signal Processing 1(1), 1-194 (2007)

27. R Harris, A variable step (VS) adaptive filter algorithm. IEEE Trans. Acoust. Speech Signal Process. ASSP-34(2), 309-316 (1986)

28. RH Kwong, EW Johnston, A variable step size LMS algorithm. IEEE Trans. Signal Process. 40(7), 1633-1644 (1992)

29. J-K Hwang, Y-P Li, Variable step-size LMS algorithm with a gradient-based weighted average. IEEE Signal Process Lett 16(12), 1043-1046 (2009)

30. H-C Huang, J Lee, A new variable step-size NLMS algorithm and its performance analysis. IEEE Trans. Signal Process. 60(4), 2055-2060 (2012)

31. T Jiang, Y Yang, YH Song, Exponential companding technique for PAPR reduction in OFDM systems. IEEE Trans. Broadcast. 51(2), 244-248 (2005)

32. E Eleftheriou, DJ Falconer, Tracking properties and steady-state performance of RLS adaptive filter algorithms. IEEE Trans. Acoust. Speech Signal Process. ASSP-34(5), 1097-1110 (1986)

33. S Haykin, Adaptive filter theory, 4th edn. (Prentice-Hall, New Jersey, 2002)

34. X Zhao, J Kivinen, P Vainikainen, K Skog, Characterization of Doppler spectra for mobile communications at $5.3 \mathrm{GHz}$. IEEE Trans. Veh. Technol $52(1), 14-23$ (2003)

35. A Mämmelä, A Kotelba, M Höyhtyä, DP Taylor, Relationship of average transmitted and received energies in adaptive transmission. IEEE Trans. Veh. Technol. 59(3), 1257-1268 (2010)

36. EO Brigham, The fast Fourier transform and its applications, 2nd ed. (PrenticeHall, New Jersey, 1988)

37. DNC Tse, $P$ Visnawath, $L$ Zheng, Diversity-multiplexing tradeoff in multipleaccess channels. IEEE Trans. Inf. Theory 50(9), 1859-1874 (2004)

38. A Goldsmith, Wireless communications (Cambridge University Press, New York, 2005)

39. PA Bello, BD Nelin, The effect of frequency selective fading on the binary error probabilities of incoherent and differentially coherent matched filter receivers. IEEE Trans. Commun. Syst. CS-11(2), 170-186 (1963)

40. S Stein, Fading channel issues in system engineering. IEEE J. Sel. Areas in Commun. SAC-5(2), 68-89 (1987)

41. Z Tian, KL Bell, HL Van Trees, Robust constrained linear receivers for CDMA wireless systems. IEEE Trans. Signal Process. 49(7), 1510-1522 (2001)

42. E Eitel, J Speidel, Decision-directed MIMO channel tracking with efficient error propagation mitigation, in IEEE 75th Vehicular Techonology Conference (VTC Spring), 2012

43. IT Ardekani, WH Abdulla, Theoretical convergence analysis of FxLMS algorithm. Signal Processing 90(12), 3046-3055 (2010) 
44. E Bjarnason, Analysis of the filtered-X LMS Algorithm. IEEE Trans. Speech Audio Process. 3(6), 504-514 (1995)

45. SM Kuo, X Kong, WS Gan, Applications of adaptive feedback active noise control system. IEEE Trans. Control Syst. Technol. 11(2), 216-220 (2003)

46. M Weiss, $U$ Timor, On the near-equivalence of envelope and square-law detection of correlated Gaussian noise. Proc. IEEE 70(5), 522-523 (1982)

47. Ml Skolnik, Introduction to radar systems, 3rd edn. (McGraw-Hill, New York, 2001)

48. WA Gardner, Learning characteristics of stochastic-gradient-descent algorithms: a general study, analysis, and critique. Signal Processing 6(2), 114-133 (1984)

49. A Chockalingam, P Dietrich, LB Milstein, RR Rao, Performance of closed-loop power control in DS-CDMA cellular systems. IEEE Trans. Veh. Technol. 47(3), 774-789 (1998)

\section{Submit your manuscript to a SpringerOpen ${ }^{\odot}$ journal and benefit from:}

- Convenient online submission

- Rigorous peer review

- Immediate publication on acceptance

Open access: articles freely available online

- High visibility within the field

- Retaining the copyright to your article

Submit your next manuscript at $\gg$ springeropen.com 